\title{
AN IMPROVED INTERFEROMETRIC CALIBRATION METHOD BASED ON INDEPENDENT PARAMETER DECOMPOSITION
}

\author{
Jun.Fan ${ }^{1,2}$, Xiaoqing.Zuo ${ }^{1, *}$, Tao.Li ${ }^{2}$, Qianfu.Chen ${ }^{2}$, Xiaomeng.Geng ${ }^{2,3}$ \\ ${ }^{1}$ Institute of Land and Resources Engineering, Kunming University of Science and Technology, Kunming, China - (funjun199401, \\ zuoxq)@163.com \\ ${ }^{2}$ Satellite Surveying and Mapping Application Center, National Administration of Surveying, Mapping and Geo-information, \\ Beijing, China - (lit, chenqf)@sasmac.cn \\ ${ }^{3}$ Beijing Satlmage Information Technology Co., Ltd, Beijing, China - gengxm0120@163.com
}

Commission III, WG III/3

KEY WORDS: Geometric calibration, Interferometric calibration, Three-dimensional reconstruction model, Sensitivity analysis, Independent parameter decomposition

\begin{abstract}
:
Interferometric SAR is sensitive to earth surface undulation. The accuracy of interferometric parameters plays a significant role in precise digital elevation model (DEM). The interferometric calibration is to obtain high-precision global DEM by calculating the interferometric parameters using ground control points (GCPs). However, interferometric parameters are always calculated jointly, making them difficult to decompose precisely. In this paper, we propose an interferometric calibration method based on independent parameter decomposition (IPD). Firstly, the parameters related to the interferometric SAR measurement are determined based on the three-dimensional reconstruction model. Secondly, the sensitivity of interferometric parameters is quantitatively analyzed after the geometric parameters are completely decomposed. Finally, each interferometric parameter is calculated based on IPD and interferometric calibration model is established. We take Weinan of Shanxi province as an example and choose 4 TerraDEM-X image pairs to carry out interferometric calibration experiment. The results show that the elevation accuracy of all SAR images is better than $2.54 \mathrm{~m}$ after interferometric calibration. Furthermore, the proposed method can obtain the accuracy of DEM products better than $2.43 \mathrm{~m}$ in the flat area and $6.97 \mathrm{~m}$ in the mountainous area, which can prove the correctness and effectiveness of the proposed IPD based interferometric calibration method. The results provide a technical basis for topographic mapping of 1:50000 and even larger scale in the flat area and mountainous area.
\end{abstract}

\section{INTRODUCTION}

The Interferometric synthetic aperture radar (InSAR) has the advantages of all-day, all-weather and high-precision, and has good application prospect in topographic mapping (Gao et al., 2017). However, the acquisition of high-precision DEM depends on design of the InSAR platform index and the calibration of interferometric parameter (González et al., 2010). Therefore, accuracy and stability of the interferometric parameter always restrict the elevation accuracy of SAR images, and interferometric calibration is an important means to improve the accuracy of DEM by improving elevation accuracy of SAR images (Gruber et al., 2016). At present, a lot of research work has been carried out for the problem of interferometric calibration both domestic and foreign, and many methods have been presented by scholars. An interferometric calibration method based on the sensitivity equation (SE) model is presented from the Germany DLR's Geudtner et al. (1999). Afterwards, in the study of the interferometric parameter calibration, the interferometric calibration method based on the SE model in squint mode is derived from the Spain UPC's Mallorqi et al. (2001), by simulation results showed that different sensitivity matrix conditions have different influences on the interferometric calibration, but there is no research on the problem of locating calibrator. After that, Wang (2003) introduced a three-dimensional reconstruction (TDR) model for the derivation of the interferometric sensitivity equation, and presented an algorithm to minimize the condition number of sensitivity matrix. Chen et al. (2014) proposed a threedimensional baseline calibration method of spaceborne InSAR, by simulation results showed that this method could complete the high-precision baseline vector calibration, but lacked the support of experimental data. Wu (2017) applied TanDEM-X to employ the interferometric calibration methods of based on TDR and SE model, which proved the universality of interferometric calibration method for spaceborne InSAR. So far, the framework of the interferometric calibration model has been established. However, the errors of most parameters are highly coupled in the process of interference, and these parameters cannot be accurately distinguished in the calculation, which lead to the inaccuracy of interferometric parameter calibration. To solve this problem, we propose an interferometric calibration method based on IPD model.

\section{THREE-DIMENSIONAL RECONSTRUCTION MODEL}

We briefly review the TDR model for InSAR which is the principle of DEM measurement. The TDR model consists of slant-range equation, Doppler equation and interferometric equation, which obtain the three-dimensional coordinates of GCPs (see Eq.(1-3)). We describe the geometrical relationship of InSAR in the geocentric space rectangular coordinates $(\mathrm{O}-$ $\mathrm{XYZ}$ ), as shown in Figure 1. We mainly focus on the Cross

\footnotetext{
Corresponding author: zuoxq@163.com
} 
Track Interferometry (XTI) mode of spaceborne InSAR (Goblirsh, 1997), and the geometrical relationship of TDR is expressed as:

$$
\begin{gathered}
\vec{P}=\vec{S}_{1}+\vec{R}_{1} \\
f_{\text {dop }}=\frac{2 \vec{v} \cdot\left(\vec{S}_{1}-\vec{P}\right)}{\lambda \cdot R_{1}} \\
\varphi=\phi+2 k \pi=\frac{2 \pi Q\left(R_{1}-R_{2}\right)}{\lambda}
\end{gathered}
$$

Where $S_{1}$ and $S_{2}$ are satellites, $\vec{P}$ is the position vector of ground target point, $R_{1}$ and $R_{2}$ are the slant-range of $S_{1}$ and $S_{2}$, $\vec{v}$ is the velocity vector, $f_{\text {dop }}$ is the Doppler frequency, $\lambda$ is the radar wavelength, $\varphi$ is the absolute phase, $\phi$ is the wrapped phase, $B$ is baseline vector, $Q$ is the antenna receiving mode.

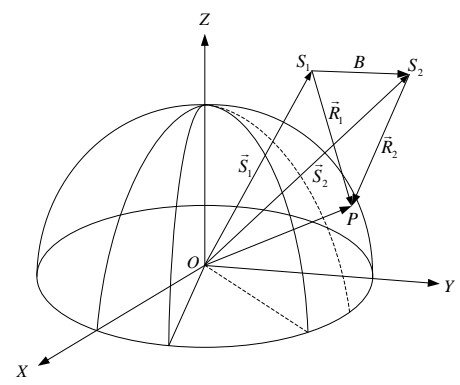

Figure 1. The geometrical relationship for spaceborne InSAR in topographic mapping

$$
\vec{P}=\vec{S}_{1}+\vec{R}_{1}=\vec{S}_{1}+R_{1} \cdot R_{p \varphi}^{-1} \cdot \hat{r}_{v \varphi}=\vec{S}_{1}+R_{1} \cdot\left[\begin{array}{lll}
\frac{\vec{v}}{|\vec{v}|} & \frac{(\vec{v} \otimes \vec{B}) \otimes \vec{v}}{|(\vec{v} \otimes \vec{B}) \otimes \vec{v}|} & \frac{(\vec{v} \otimes \vec{B})}{|(\vec{v} \otimes \vec{B})|}
\end{array}\right]\left[\begin{array}{l}
\frac{\lambda f_{\text {dop }}}{2|\vec{v}|} \\
\frac{R_{1}}{2\left|b_{p v}\right|}\left[1+\frac{B^{2}}{R_{1}^{2}}-\left(1-\frac{\lambda \varphi}{2 \pi Q R_{1}}\right)^{2}\right]-\frac{b_{v} r_{v}}{\left|b_{p v}\right|} \\
\pm \sqrt{1-r_{v}^{2}-r_{p}^{2}}
\end{array}\right]
$$

Where $R_{v q}^{-1}$ is the transformation matrix, $b_{v}$ is the component of baseline in the velocity direction, and $b_{p v}$ is the component of baseline in the direction perpendicular to the velocity.

In Equation (6), observations of the TDR model are satellite position, satellite velocity, the slant-range, Doppler frequency, interferometric phase, baseline vector. The satellite position, satellite velocity, slant-range, Doppler frequency are calibrated in geometric calibration (Sadelain et al., 2011), which can improve positioning accuracy of SAR image, leaving only the interferometric phase as well as the baseline vector are calibrated by the interferometric calibration method proposed in this paper, which can improve elevation accuracy of SAR images. In this way, high-precision DEM can be obtained.

\section{INTERFEROMETRIC SENSITIVITY ANALYSES}

We analyze the sensitivity of the interferometric phase and baseline vector under the guarantee of geometric accuracy, and quantitatively obtain the influence of interferometric parameter on elevation error.
According to the geometrical relationship of interferometric SAR, the target point coordinates are expressed in the O-XYZ (Knedlik et al., 2000).

$$
\vec{P}=\vec{S}_{1}+\vec{R}_{1}=\vec{S}_{1}+R_{1} \cdot \hat{r}
$$

Where $\vec{R}_{1}$ is the look vector, $\hat{r}$ is the unit look vector. In Equation (4), the look vector decomposition (LVD) method is generally used to obtain the target point coordinates (Mallorqui et al., 2000), we usually convert the look vector to the unit look vector in the calculation, and unit look vector needs to be decomposed into three axes of $\mathrm{V}, \mathrm{P}$ and $\mathrm{Q}$ in the mobile coordinate system (VPQ). VPQ is based on phase canter of main antenna as the origin of coordinate, the velocity vector direction is $\mathrm{V}$ axis, velocity and baseline cross product direction are $\mathrm{Q}$ axis, and $\mathrm{V}, \mathrm{P}, \mathrm{Q}$ meet the right hand coordinate system. $r_{v}, r_{p}$ and $r_{p}$ are considered as components of the unit look vector on the $\mathrm{V}, \mathrm{P}$, and $\mathrm{Q}$, respectively, $\hat{r}$ can be written as follows:

$$
\hat{\boldsymbol{r}}=\left[\begin{array}{l}
r_{v} \\
r_{p} \\
r_{q}
\end{array}\right]
$$

LVD method converts the look vector from the VPQ to the O$\mathrm{XYZ}$, which can obtain the three-dimensional coordinates of the target point, as shown in Eq.(6).

\subsection{Interferometric phase error sensitivity analysis}

The main component of interferometric phase error is absolute phase deviation in this paper. The DEM elevation error caused by the interferometric phase error is denoted as:

$$
\Delta h=H o A \frac{\Delta \varphi}{2 \pi}
$$

Where HoA is the height of ambiguity. The TanDEM-X mission adopt two setting. During the first mission with height of ambiguity is about 40-55 m, while for the second year height of ambiguity is about $35 \mathrm{~m}$ (Rossi et al., 2012). When HoA=35 $\mathrm{m}$, the phase error of $10^{\circ}$ is introduced into the elevation error of about $0.97 \mathrm{~m}$; When $\mathrm{HoA}=55 \mathrm{~m}$, the phase error of $10^{\circ}$ is introduced into the elevation error of about $1.54 \mathrm{~m}$. We assume that the interferometric phase error which range from $0^{\circ}$ to $10^{\circ}$, and assess the elevation error using TDR model. As shown in Figure 2, we found that elevation error of about 1.42 caused by phase error of $10^{\circ}$, and with the increase of interferometric phase error, the elevation error increase linearly. If the 1:50000 scale topographic mapping are to be completed, the elevation precision of the hilly area needs to be $5 \mathrm{~m}$, and here the effect of the phase error has reached about $28.4 \%$. Therefore, the interferometric phase error is an important error source in the topographic mapping of InSAR. 


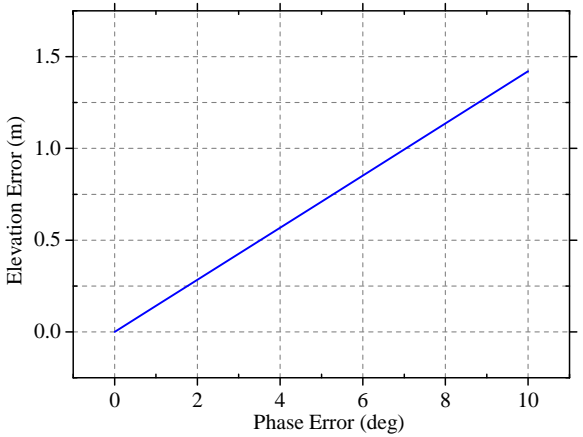

Figure 2. Sensitivity analysis of interferometric phase

\subsection{Baseline vector sensitivity analysis}

In the topographic mapping of InSAR, the baseline vector directly influences height of ambiguity and elevation error (González et al., 2010). The baseline is a three-dimensional vector, in order to express the baseline parameters more conveniently, the Track-Cross-Normal (TCN) coordinate system is generally adopted. The master antenna phase center as the origin of coordinates, $\mathrm{N}$ axis is from the main antenna phase canter pointing to the geocenter, the $\mathrm{N}$ axis and the velocity vector cross product direction is the positive direction of the $\mathrm{C}$ axis, $\mathrm{T}, \mathrm{C}$ and $\mathrm{N}$ meet the right hand coordinate system. The baseline vector is decomposed into initial baseline and timerelated baseline rate in the TCN. This paper mainly focuses on the cross track interferometry mode of InSAR. Because of the baseline error component in $\mathrm{T}$ direction, also known as along track direction, there is sufficiently eliminated during slave image registration, we do not consider the $\mathrm{T}$ component (Krieger et al., 2007). Therefore, the baseline vector can be expressed as:

$$
\left\{\begin{array}{l}
B_{t}=0 \\
B_{c}=b_{c 0}+t \cdot b_{c v} \\
B_{n}=b_{n 0}+t \cdot b_{n v}
\end{array}\right.
$$

Because of the influence of baseline error on DEM elevation accuracy is relatively complex, we analyze the influence of the baseline component $b_{c 0}, b_{c v}, b_{n 0}$ and $b_{n v}$ on the elevation accuracy. As shown in Figure3, baseline component sensitivity analysis shows that $1 \mathrm{~mm}$ error of the initial baseline under $\mathrm{C}$ and $\mathrm{N}$ direction cause $1.2 \mathrm{~m}$ and $-1.6 \mathrm{~m}$ elevation error, respectively, and $0.1 \mathrm{~mm} / \mathrm{s}$ error of the baseline rate under $\mathrm{C}$ and $\mathrm{N}$ direction cause $0.5 \mathrm{~m}$ and $-0.6 \mathrm{~m}$ elevation error, respectively. From the above analysis, it is clear that the baseline error of millimeter level can cause the elevation error of meter level, and the baseline rate error of submillimeter level can cause the elevation error of meter level. In addition, compared with the initial baseline error, the baseline rate error is more sensitive to the accuracy of the elevation; Compared with the baseline vector error in the $\mathrm{C}$ direction, the $\mathrm{N}$ direction is more sensitive to the accuracy of the elevation. Therefore, the baseline vector is also an important error source in the topographic mapping of InSAR.
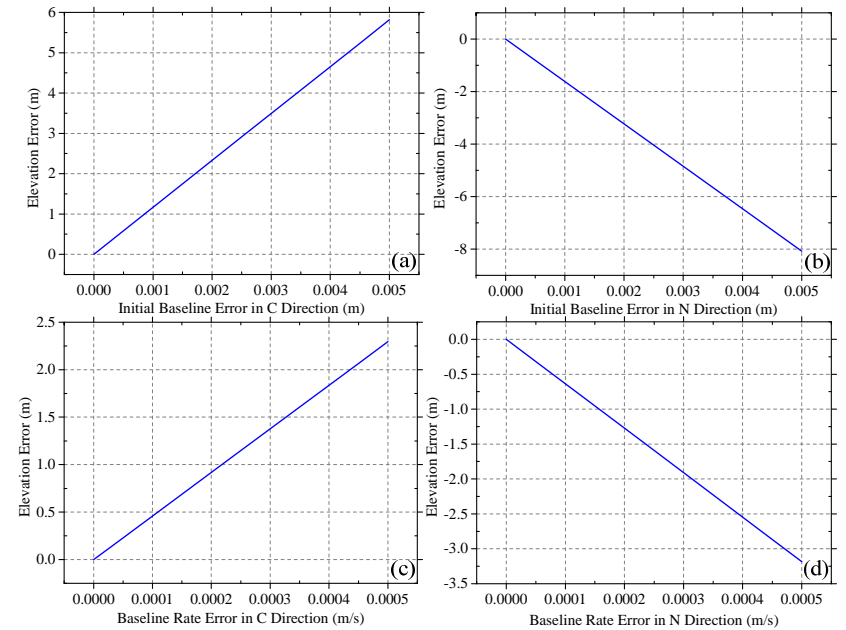

Figure 3. Sensitivity analysis of baseline component

\section{INSAR INTERFEROMETRIC CALIBRATION MODEL}

The establishment of the interferometric calibration model is based on the different transfer characteristics of the interferometric phase and the baseline vector to the elevation error (Rizzoli, 2012). In this paper, we first calibrate the phase error and then calibrate the baseline vector error. In order to ensure that interferometric parameters are completely independent in the calculation, the interferometric phase error is obtained by calculating the fixed deviation between absolute phase and unwrapped phase and flat phase (Hanssen, 2001).

First of all, the absolute phase of each point is obtained by the GCPs. Then, the unwrapped phase and flat phase are compensated according to the absolute phase. Finally, the fixed deviation should be compensated for the phase data of SAR image. In this way, the interferometric phase error of each image can be obtained as follows:

$$
\Delta \varphi=\frac{4 \pi\left(R_{1}-R_{2}\right)}{\lambda}-\varphi_{u n w}-\varphi_{f l a}
$$

Where $\Delta \varphi$ is the interferometric phase error, $\varphi_{\text {unw }}$ is the unwrapped phase, $\varphi_{f a}$ is the flat phase.

Baseline vector calibration begins with the TDR model of InSAR. Then the sensitivity equations are derived based on the partial derivatives of interferometric parameters. Finally, the correction of interferometric parameter is calculated based on the least square (LS) method. To improve the accuracy of interferometric parameter, we adopt the iterative method. The root mean square (RMS) of the coordinate difference before and after iteration is defined as the iterative precision, the iteration stop when iteration precision is less than the specified threshold. To summarize, interferometric parameters with high-precision are obtained. Flow chart of interferometric calibration is shown in Figure 4. 


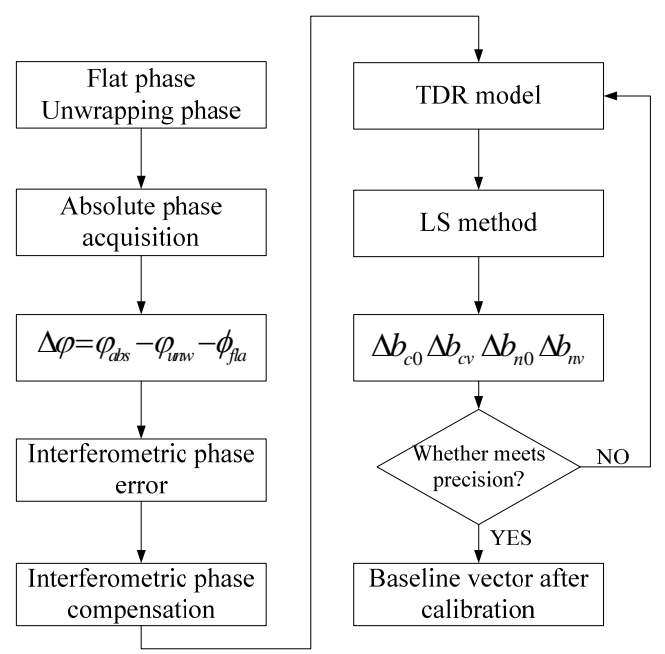

Figure 4. Flow chart of interferometric calibration
The TDR equations can be derived by the TDR model, which can be denoted as:

$$
\left[\begin{array}{l}
F_{x} \\
F_{y} \\
F_{z}
\end{array}\right]=\left[\begin{array}{l}
S_{x} \\
S_{y} \\
S_{z}
\end{array}\right]-\left[\begin{array}{l}
P_{x} \\
P_{y} \\
P_{z}
\end{array}\right]+R_{1}\left[\begin{array}{lll}
a_{11} & a_{12} & a_{13} \\
a_{21} & a_{22} & a_{23} \\
a_{31} & a_{32} & a_{33}
\end{array}\right]\left[\begin{array}{l}
r_{v} \\
r_{p} \\
r_{q}
\end{array}\right]
$$

Where $\left[\begin{array}{lll}S_{x} & S_{y} & S_{z}\end{array}\right]^{\mathrm{T}}$ is the coordinate of the master antenna phase center, $\left[\begin{array}{lll}P_{x} & P_{y} & P_{z}\end{array}\right]^{\mathrm{T}}$ is the coordinate of target, $R_{1}$ is the slant range between the target and the satellite, $\left[\begin{array}{lll}a_{11} & a_{12} & a_{13} \\ a_{21} & a_{22} & a_{23} \\ a_{31} & a_{32} & a_{33}\end{array}\right]$ is the transformation matrix.

The partial derivative of the baseline vector was calculated based on the TDR equation, and the interferometric sensitivity equation was established (see Eq.(11)).

$$
\left[\begin{array}{l}
\frac{\partial F_{x}}{\partial X} \\
\frac{\partial F_{y}}{\partial X} \\
\frac{\partial F_{z}}{\partial X}
\end{array}\right]=\left[\begin{array}{l}
\frac{\partial S_{x}}{\partial X} \\
\frac{\partial S_{y}}{\partial X} \\
\frac{\partial S_{z}}{\partial X}
\end{array}\right]+\frac{\partial R_{1}}{\partial X}\left[\begin{array}{lll}
a_{11} & a_{12} & a_{13} \\
a_{21} & a_{22} & a_{23} \\
a_{31} & a_{32} & a_{33}
\end{array}\right]\left[\begin{array}{l}
r_{v} \\
r_{p} \\
r_{q}
\end{array}\right]+R_{1}\left\{\left[\begin{array}{lll}
\frac{\partial a_{11}}{\partial X} & \frac{\partial a_{12}}{\partial X} & \frac{\partial a_{13}}{\partial X} \\
\frac{\partial a_{21}}{\partial X} & \frac{\partial a_{22}}{\partial X} & \frac{\partial a_{23}}{\partial X} \\
\frac{\partial a_{31}}{\partial X} & \frac{\partial a_{32}}{\partial X} & \frac{\partial a_{33}}{\partial X}
\end{array}\right]\left[\begin{array}{l}
r_{v} \\
r_{p} \\
r_{q}
\end{array}\right]+\left[\begin{array}{lll}
a_{11} & a_{12} & a_{13} \\
a_{21} & a_{22} & a_{23} \\
a_{31} & a_{32} & a_{33}
\end{array}\right]\left[\begin{array}{l}
\frac{\partial r_{v}}{\partial X} \\
\frac{\partial r_{p}}{\partial X} \\
\frac{\partial r_{q}}{\partial X}
\end{array}\right]\right\}
$$

The error equation is established as follows:

$$
V_{B}=\left[\begin{array}{llll}
\frac{\partial F_{x 1}}{\partial b_{c 0}} & \frac{\partial F_{x 1}}{\partial b_{c v}} & \frac{\partial F_{x 1}}{\partial b_{n 0}} & \frac{\partial F_{x 1}}{\partial b_{n v}} \\
\frac{\partial F_{y 1}}{\partial b_{c 0}} & \frac{\partial F_{y 1}}{\partial b_{c v}} & \frac{\partial F_{y 1}}{\partial b_{n 0}} & \frac{\partial F_{x 1}}{\partial b_{n v}} \\
\frac{\partial F_{z 1}}{\partial b_{c 0}} & \frac{\partial F_{z 1}}{\partial b_{c v}} & \frac{\partial F_{z 1}}{\partial b_{n 0}} & \frac{\partial F_{x 1}}{\partial b_{n v}} \\
\ldots & \ldots & \ldots & \ldots \\
\frac{\partial F_{x n}}{\partial b_{c 0}} & \frac{\partial F_{x n}}{\partial b_{c v}} & \frac{\partial F_{x n}}{\partial b_{n 0}} & \frac{\partial F_{x n}}{\partial b_{n v}} \\
\frac{\partial F_{y n}}{\partial b_{c 0}} & \frac{\partial F_{y n}}{\partial b_{c v}} & \frac{\partial F_{y n}}{\partial b_{n 0}} & \frac{\partial F_{y n}}{\partial b_{n v}} \\
\frac{\partial F_{z n}}{\partial b_{c 0}} & \frac{\partial F_{z n}}{\partial b_{c v}} & \frac{\partial F_{z n}}{\partial b_{n 0}} & \frac{\partial F_{z n}}{\partial b_{n v}}
\end{array}\right]\left[\begin{array}{c}
\Delta b_{c 0} \\
\Delta b_{c v} \\
\Delta b_{n 0} \\
\Delta b_{n v}
\end{array}\right]+\left[\begin{array}{c}
\left(F_{x 1}\right)-F_{x 1} \\
\left(F_{y 1}\right)-F_{y 1} \\
\left(F_{z 1}\right)-F_{z 1} \\
\cdots \\
\ldots \\
\left(F_{x n}\right)-F_{x n} \\
\left(F_{y n}\right)-F_{y n} \\
\left(F_{z n}\right)-F_{z n}
\end{array}\right]
$$

Where $\Delta b_{c 0}$ and $\Delta b_{c v}$ denote the correction of the initial baseline and the baseline rate in the $\mathrm{C}$ direction, respectively; $\Delta b_{n 0}$ and $\Delta b_{n v}$ denote the correction of the initial baseline and the baseline rate in the $\mathrm{N}$ direction, respectively. The observation error can be calculated as follow:

$$
\left[\begin{array}{lll}
\left(F_{x 1}\right) \cdots & \left(F_{z n}\right)
\end{array}\right]^{\mathrm{T}}=\left[\begin{array}{lll}
P_{x 1}^{0}-P_{x 1} & \cdots & P_{z n}^{0}-P_{z n}
\end{array}\right]^{\mathrm{T}}
$$

where $\left[\begin{array}{lll}P_{x i}^{0} & P_{y i}^{0} & P_{z i}^{0}\end{array}\right]^{\mathrm{T}}$ is the three-dimensional reconstruction coordinates of the targets, $\left[\begin{array}{lll}P_{x i} & P_{y i} & P_{z i}\end{array}\right]^{\mathrm{T}}$ are the known GCPs coordinates. $\left[\begin{array}{lll}F_{x 1} & \cdots & F_{z n}\end{array}\right]^{\mathrm{T}}=0$ is the true value of observation.

In the process of interferometric calibration, the accuracy of observation is usually different due to different observation conditions, and the introduction of error is different. Since coherence coefficient is an important criterion to evaluate SAR image quality. Therefore, the coherence coefficient of GCPs is weighted in this paper (Mao et al., 2013), the objective function is expressed as:

$$
J_{\min }=V^{T} W V
$$

Where $W$ is the diagonal matrix, it can be written as Eq.(15):

$$
W=\left[\begin{array}{cccc}
w_{1} & 0 & \cdots & 0 \\
0 & w_{2} & & \vdots \\
\vdots & & \ddots & 0 \\
0 & \cdots & 0 & w_{n}
\end{array}\right]
$$

Where $w_{i}$ is the coherence coefficient for each GCP. Given the initial value of interferometric parameters, the correction of the interferometric parameters is calculated as follows.

$$
\Delta X=\left(A^{T} W A\right)^{-1} A^{T} W l
$$

The interferometric parameters are calculated after calibration, as shown in Eq.(17).

$$
X=X_{0}+\Delta X
$$

\section{EXPERIMENTAL AND ANALYSIS}

\subsection{Experimental Data}

The study area is located in Weinan city of Shanxi province is covering $34.519^{\circ} \sim 35.546^{\circ}$, $109.147^{\circ} \sim 110.005^{\circ}$, the east-west range is about $63 \mathrm{~km}$, and the north-south range is about $108 \mathrm{~km}$. This area is highly undulated, the height difference between the northern mountainous and southern plain is about $1000 \mathrm{~m}$, the 
geographical location is shown in Figure 5. Road intersection, corner of house and other feature points are selected as GCPs in this paper. The GCPs are acquired by the differential global positioning system (DGPS).

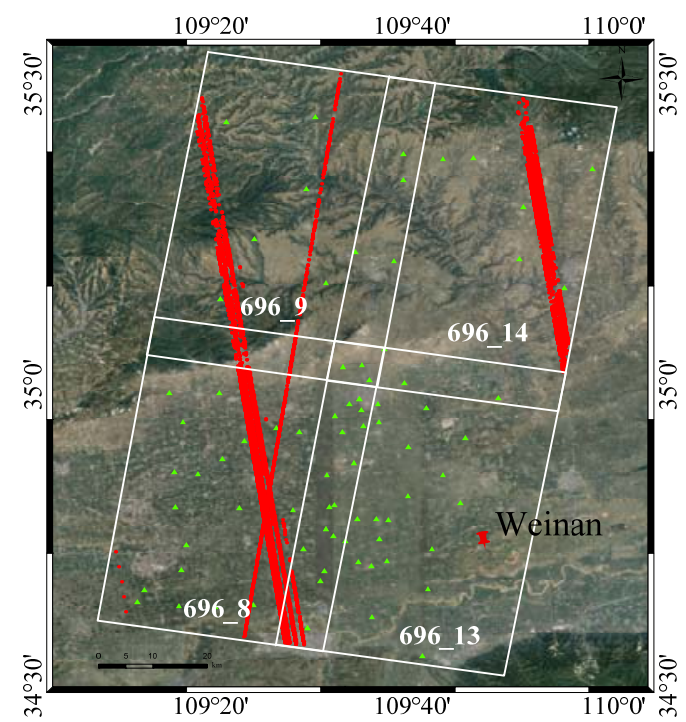

Figure 5. Geographical position and point's distribution of study region, where the green triangles are the GCPs and the red dots are ICESat data.

We choose 4 TanDEM-X/TerraSAR-X image pairs to carry out interferometric calibration experiment. The images were acquired on September 23, 2013 and September 25, 2013. We choose the $90 \mathrm{~m}$ SRTM as external reference data. In Table 1 presents statistics on the parameters of experimental images.

\begin{tabular}{ccc}
\hline Image ID & $\begin{array}{c}\text { Initial Baseline in TCN } \\
\text { Direction (m) }\end{array}$ & $\begin{array}{c}\text { Time-Related } \\
\text { Baseline Rate of in } \\
\text { TCN Direction }(\mathrm{m} / \mathrm{s})\end{array}$ \\
\hline 696_8 & $0,233.7248,-51.8344$ & $0,0.1969,0.2896$ \\
$696 \_9$ & $0,232.3390,-53.8600$ & $0,0.1990,0.2891$ \\
696_13 & $0,184.7189,-94.2540$ & $0,0.1664,0.2696$ \\
696_14 & $0,183.5485,-96.1386$ & $0,0.1680,0.2688$ \\
\hline
\end{tabular}

Table 1. Parameters of experimental images

\subsection{Result and Analysis}

Under the condition of ensuring geometric accuracy (Deng et al., 2017), the elevation of GCPs is calculated based on the TDR model, and the accuracy is assessed with the elevation of the known GCPs. In Table 2 lists the experimental results before interferometric calibration.

\begin{tabular}{cc}
\hline Image ID & Elevation accuracy $(\mathrm{m})$ \\
\hline 696_8 & 20.016 \\
696_9 & 21.591 \\
696_13 & 9.444 \\
696_14 & 8.925 \\
\hline
\end{tabular}

Table 2. Elevation accuracy of SAR images before interferometric calibration

Table 2 shows that there are significant differences in the elevation accuracy of different orbit images. For example, the elevation accuracy of the 696_13 and 696_14 image is lower than $9.44 \mathrm{~m}$, while elevation accuracy of 696_8 and 696_9 image is lower than $21.59 \mathrm{~m}$ in the same orbit. Firstly, due to the limitation of the terrain, the accuracy of the GCP itself is reduced; secondly, there is a residual error in the geometric calibration, which leads to the introduction of geometric errors in the interferometric calibration.

Considering that the interferometric phase error may be introduced when the baseline error is calibrated at the beginning, afterwards, the elevation error is increased when the interferometric phase error is calibrated. Therefore, we first calibrate the interference phase error. Table 3 lists statistics on the experimental results after interferometric phase calibration.

\begin{tabular}{ccc}
\hline Image ID & $\begin{array}{c}\text { Interferometric } \\
\text { Phase Error (deg) }\end{array}$ & $\begin{array}{c}\text { Elevation Accuracy } \\
(\mathrm{m})\end{array}$ \\
\hline 696_8 & -120.768 & 2.057 \\
696_9 & -127.059 & 2.848 \\
696_13 & -50.099 & 2.726 \\
696_14 & -52.105 & 2.312 \\
\hline
\end{tabular}

Table 3. Evaluation accuracy after interferometric phase calibration

As Table 3 shows, all images elevation precision is better than $2.85 \mathrm{~m}$ after the interferometric phase error calibration. On this basis, we calibrate the baseline vector based on the calibration method which is different from the phase error. Table 4 lists the experimental results after baseline vector calibration.

\begin{tabular}{cccccc}
\hline \multirow{2}{*}{$\begin{array}{c}\text { Image } \\
\text { ID }\end{array}$} & \multicolumn{3}{c}{ The Correction Baseline Component } & $\begin{array}{r}\text { Elevation } \\
\text { Accuracy }\end{array}$ \\
\cline { 2 - 6 } & $\Delta b_{c 0}(\mathrm{~m})$ & $\Delta b_{n 0}(\mathrm{~m})$ & $\Delta b_{c v}(\mathrm{~m} / \mathrm{s})$ & $\Delta b_{n v}(\mathrm{~m} / \mathrm{s})$ & $(\mathrm{m})$ \\
\hline $696 \_8$ & 0.0597 & -0.0473 & -0.0092 & 0.0072 & 1.469 \\
$696 \_9$ & 0.0649 & -0.0516 & 0.0008 & -0.0007 & 1.506 \\
$696 \_13$ & -0.0552 & 0.0420 & 0.0057 & -0.0043 & 2.535 \\
$696 \_14$ & -0.0671 & 0.0507 & -0.0174 & 0.0128 & 1.768 \\
\hline
\end{tabular}

Table 4. Evaluation accuracy after baseline calibration

As Table 4 shows, all images elevation precision is better than $2.54 \mathrm{~m}$ after baseline vector error calibration. Compared with the elevation accuracy before interferometric calibration, the elevation accuracy of all SAR images is obviously improved.

In this paper, the main error sources in InSAR system are decomposed by the proposed method of interferometric calibration method with IPD. Meanwhile, these factors are the main sources of DEM product errors. Furthermore, root mean square error (RMSE) of DEM products are assessed by using the shuttle radar topography mission (SRTM) DEM and the Ice, Cloud, and Elevation Satellite (ICESat) data before and after interferometric calibration (Gruber et al., 2012). Table 5 shows the results of DEM products assessment.

\begin{tabular}{ccccc}
\hline \multirow{2}{*}{$\begin{array}{c}\text { Image } \\
\text { ID }\end{array}$} & \multicolumn{2}{c}{$\begin{array}{c}\text { RMSE of } \\
\text { Uncalibrated } \\
\text { DEM }\end{array}$} & \multicolumn{2}{c}{$\begin{array}{c}\text { RMSE of } \\
\text { Calibrated DEM }\end{array}$} \\
\cline { 2 - 5 } & SRTM & ICESat & SRTM & ICESat \\
\hline $696 \_8$ & 10.482 & 11.671 & 0.626 & 1.154 \\
$696 \_9$ & 14.132 & 12.652 & 6.972 & 3.376 \\
$696 \_13$ & 10.700 & 10.374 & 1.266 & 2.428 \\
$696 \_14$ & 11.020 & 10.391 & 4.864 & 2.528 \\
\hline
\end{tabular}

Table 5. DEM precision assessment (in unit: $\mathrm{m}$ ) 
As shown in Figure 5, 696_8 and 696_13 are located in the flat area as well as 696_9 and 696_14 are located in the mountainous area. In Table 5 presents the RMSEs obtained using SRTM are lower than $10.70 \mathrm{~m}$ in the flat area and $14.13 \mathrm{~m}$ in the mountainous area for uncalibrated DEM. And RMSEs from ICESat are lower than $11.67 \mathrm{~m}$ in the flat area and $12.65 \mathrm{~m}$ in the mountainous area for uncalibrated DEM. RMSEs obtained using SRTM are better than $1.27 \mathrm{~m}$ in the flat area and $6.97 \mathrm{~m}$ in the mountainous for calibrated DEM. And RMSEs from ICESat are better than $2.43 \mathrm{~m}$ in the flat area and $3.38 \mathrm{~m}$ in the mountainous area for calibrated DEM. Therefore, the proposed interferometric calibration method can improve the accuracy of DEM products both in the flat and mountainous area. However, the precision of obtaining DEM products in the flat area is always better than that in the mountainous area, the main reason is that the mountainous terrain is undulating, and the vegetation is flourishing as well as the coherence is low. There is a certain influence on the interferometric results, which leads to the elevation error of the area is large, and the error distribution is relatively discrete.

\section{CONCLUSION}

At present, with the successive launch of the domestic SAR satellites, it is urgent to further study the interferometric calibration technology for spaceborne InSAR, and improve the ability of obtaining high-precision DEM for domestic spaceborne InSAR system. Because of the research of interferometric calibration is generally carried out from the airborne InSAR both domestic and foreign, and it is gradually extended to the platform for spaceborne InSAR. This paper mainly focuses on the problem of parameter coupling for traditional interferometric calibration model, we propose a improved method of interferometric calibration based on the IDP model for spaceborne InSAR. We employ two independent calibration methods to calibrate the phase error and baseline vector errors, respectively, and choose TanDEM-X/TerraSAR$\mathrm{X}$ image pairs to carry out interferometric calibration experiment. The results show that the elevation accuracy of SAR images is better than $2.54 \mathrm{~m}$. The accuracy of DEM products was assessed based on the external SRTM DEM and ICESat data, and the elevation accuracy of DEM products is better than $2.43 \mathrm{~m}$ in the flat area as well as $6.97 \mathrm{~m}$ in mountainous area after the calibration. Therefore, the method of interferometric calibration proposed can improve the accuracy of DEM products, which prove that the method is effective and feasible.

In conclusion, we propose this method of interferometric calibration based on IDP model, and hope to provide some technical reference for spaceborne InSAR in China, especially provide certain technical support for the subsequent satellite onorbit calibration. In addition, there are some problems that need to be studied in this paper, the number and distribution of GCPs are not discussed, particularly how to reduce the number of GCPs and achieve the interferometric calibration of sparse GCPs. Furthermore, because of the lack of high-precision corner reflector in the experiment, we did not verify the onorbit calibration of the satellite. Therefore, the follow-up research work will mainly focus on these two aspects, and hope to provide more favorable support for the interferometric calibration method based on IDP in this paper.

\section{ACKNOWLEDGEMENTS}

This work was supported by National Key R\&D Programme of China (NO: 2017YFB0502700), Civilian Space Programme of China (DO: D010102), National Basic Surveying and Mapping Science and Technology Plan (NO: 2018KJ0204/2018KJ0304). The authors would like to thank German Aerospace Center (DLR) for providing TanDEM-X data (No: CAL_VAL6993) and National Aeronautics and Space Administration (NASA) for providing ICESat data.

\section{REFERENCES}

Gao, X., Liu, Y., \& Li, T. 2017. High Precision DEM Generation Algorithm Based on InSAR Multi-Look Iteration. Remote Sensing, 9(7), pp. 741.

González, J.H., Bachmann, M., \& Krieger, G. 2010. Development of the TanDEM-X calibration concept: Analysis of systematic errors. IEEE Transactions on GeoScience And Remote Sensing, 48(2), pp. 716-726.

Gruber, A., Wessel, B., Martone, M., \& Roth, A. 2016. The TanDEM-X DEM Mosaicking: Fusion of Multiple Acquisitions Using InSAR Quality Parameters. IEEE Journal of Selected Topics in Applied Earth Observations and Remote Sensing, 9(3), pp. 1047-1057.

Geudtner, D., \& Zink, M. 1999. Interferometric calibration of the X-SAR system on the Shuttle Radar Topography Mission(SRTM/X-SAR). In: International Airborne Remote Sensing Conference and Exhibition, 4 th/21 st Canadian Symposium on Remote Sensing, Ottawa, Canada.

Mallorqui, J.J., Bara, M., \& Broquetas, A. 2000. Calibration requirements for airborne SAR interferometry. In: Europto Remote Sensing, 32, pp. 267-278.

Wang, Y. P. 2011, Studies on calibration model and algorithm for airborne interferoemtric SAR. Institute of Electrics, Chinese Academy of Sciences.

Chen, G., Tang, X.T., 2014. A three-dimensional baseline calibration method of spaceborne InSAR. Geomatics and Information Science of Wuhan University, 39(1), pp. 37-41.

Wu, D.Q., 2017, Modeling and methodology for airborne and spaceborne InSAR calibration. Southwest Jiaotong University.

Goblirsch, W. 1997. The exact solution of the imaging equations for crosstrack interferometers. In: Geoscience and Remote Sensing, IGARSS '97. Remote Sensing - A Scientific Vision for Sustainable Development. IEEE International, 1, pp. 439-441.

Sadelain, M., \& Rivière, I. 2011. In-Orbit Calibration of the TanDEM-X System. IEEE Transactions on Applied Superconductivity, 24(8), pp. 1-4.

Knedlik, S., \& Loffeld, O. 2000. Analysis of different maximum a posteriori estimation approaches for interferometric parameter calibration. In: Geoscience and Remote Sensing Symposium, Proceedings. IGARSS. IEEE International, 2, pp. 773-775. 
Rossi, C., Rodriguez Gonzalez, F., \& Fritz, 2012. TanDEM-X calibrated Raw DEM generation. ISPRS Journal of Photogrammetry and Remote Sensing, 73(9), pp. 12-20.

Krieger, G., Moreira, A., \& Fiedler, H. 2007. TanDEM-X: A satellite formation for high-resolution SAR interferometry. IEEE Transactions on GeoScience And Remote Sensing, 45(11), pp. 3317-3341.

Rizzoli P, B.B., Kraus T, et al. 2012. Relative height error analysis of TanDEM-X elevation data. ISPRS Journal of Photogrammetry and Remote Sensing, 73(6), pp. 30-38.

Hanssen, R.F. 2001. Radar Interferometry: Data Interpretation and Error Analysis.

Krieger, G., Zink, M., Schrank, D., \& Schwerdt, M. 2012. Bistatic system and baseline calibration in TanDEM-X to ensure the global digital elevation model quality. ISPRS Journal of Photogrammetry and Remote Sensing, 73(3), pp. 311.

Mao, Y., Xiang, M., Han, Y., \& Gao, W. 2013. Weighted joint calibration for interferometric SAR. Journal of Systems Engineering and Electronics, 24(5), pp. 761-771.

Deng, M., Zhang, G., and Zhao, R., 2017. Improvement of Gaofen-3 absolute positioning accuracy based on crosscalibration. Sensors, 17(12), pp. 2903.

Gruber, A., Wessel, B., \& Huber, M. 2012. Operational TanDEM-X DEM calibration and first validation results. ISPRS Journal of Photogrammetry and Remote Sensing, 73(6), pp. 3949. 